\title{
The medial temporal lobe and visual working memory: Comparisons across tasks, delays, and visual similarity
}

\author{
YOUSSEF EZZYAT \\ University of Pennsylvania, Philadelphia, Pennsylvania \\ AND \\ INGRID R. OLSON \\ Temple University, Philadelphia, Pennsylvania
}

\begin{abstract}
Whether the hippocampus and medial temporal lobe (MTL) play any important role in visual working memory is a relatively new and controversial research question. The primary goal of this study was to assess working memory for faces over very short delays in patients with MTL damage. Patients and matched controls were required to remember one face that was parametrically morphed to be more or less similar to a probe face, over either a 1- or an 8-sec delay. Memory was assessed using both forced choice and old-new recognition tasks. The results show that MTL damage impairs both speed and accuracy of visual working memory across tasks. We speculate that the hippocampus is generally necessary for memory encoding.
\end{abstract}

Although it has long been accepted that the medial temporal lobe (MTL), including cortical areas and the hippocampus, is vital for memory formation, many questions still remain about how exactly these areas contribute to memory encoding, consolidation, and retrieval. Several findings in the rodent literature have shown that the hippocampus is critically involved in the encoding of new information into memory. For example, when NMDA receptors in the hippocampus are blocked, encoding is impaired on a paired-associate task, whereas retrieval is spared (Day, Langston, \& Morris, 2003). In addition, lesions to the rat hippocampus have been shown to produce deficits in encoding spatial information in a radial-arm maze task (Jarrard, Davidson, \& Bowring, 2004). There is also suggestive evidence from human neuroimaging pointing to an important role for the hippocampus in memory encoding. Several studies have reported that anterior portions of the hippocampus are activated when participants encode novel objects or novel associations into memory, whereas posterior portions of the hippocampus are activated during memory retrieval (Davachi, Mitchell, \& Wagner, 2003; Dobbins, Rice, Wagner, \& Schacter, 2003; Düzel et al., 2003; Eldridge, Knowlton, Furmanski, Bookheimer, \& Engel, 2000; Jackson \& Schacter, 2004; Lepage, Habib, \& Tulving, 1998; Pihlajamäki et al., 2003; Pihlajamäki et al., 2004; Preston, Shrager, Dudukovic, \& Gabrieli, 2004; Small et al., 2001; Stark \& Squire, 2001; Strange, Fletcher, Henson, Friston, \& Dolan, 1999; Strange, Otten, Josephs, Rugg, \& Dolan, 2002; Zeineh, Engel, Thompson, \& Bookheimer, 2003). However, the opposite has also been observed in several studies (for a review, see Schacter \& Wagner, 1999).

As evidenced by the lack of convergence in the imaging literature, questions remain about the specific encoding mechanisms in the human hippocampus and MTL. There has been a strong effort to dissect the differences between recall and familiarity, and especially to attribute these processes to specific MTL subregions (O'Reilly \& Norman, 2002; Yonelinas, 2002; Yonelinas et al., 2002). In humans, these results have been incorporated into a dualprocess computational model of MTL mnemonic function (Norman \& O'Reilly, 2003; O'Reilly \& McClelland, 1994; O'Reilly \& Norman, 2002; Rolls, 1996). In rodents, lesion studies of various types have also attempted to provide a concise description of MTL encoding processes, focusing on the hippocampus (Kesner \& Hopkins, 2006).

Although it would seem to be closely related to questions of memory encoding, the contribution of MTL to working memory has generally received relatively little attention. Recently, researchers have begun to explore working memory in MTL amnesic patients and have found deficits with a variety of stimuli (Olson, Moore, Stark, \& Chatterjee, 2006) and tasks (Olson, Page, Moore, Chatterjee, \& Verfaellie, 2006). These findings join neuroimaging research that has shown MTL activations during working memory tasks (Ranganath, Cohen, \& Brozinsky, 2005). This work has lent credibility to the idea that MTL is not exclusively involved in long-term memory (LTM). The fact that the hippocampus and MTL cortex are known to be important for long-term and episodic memory raises 

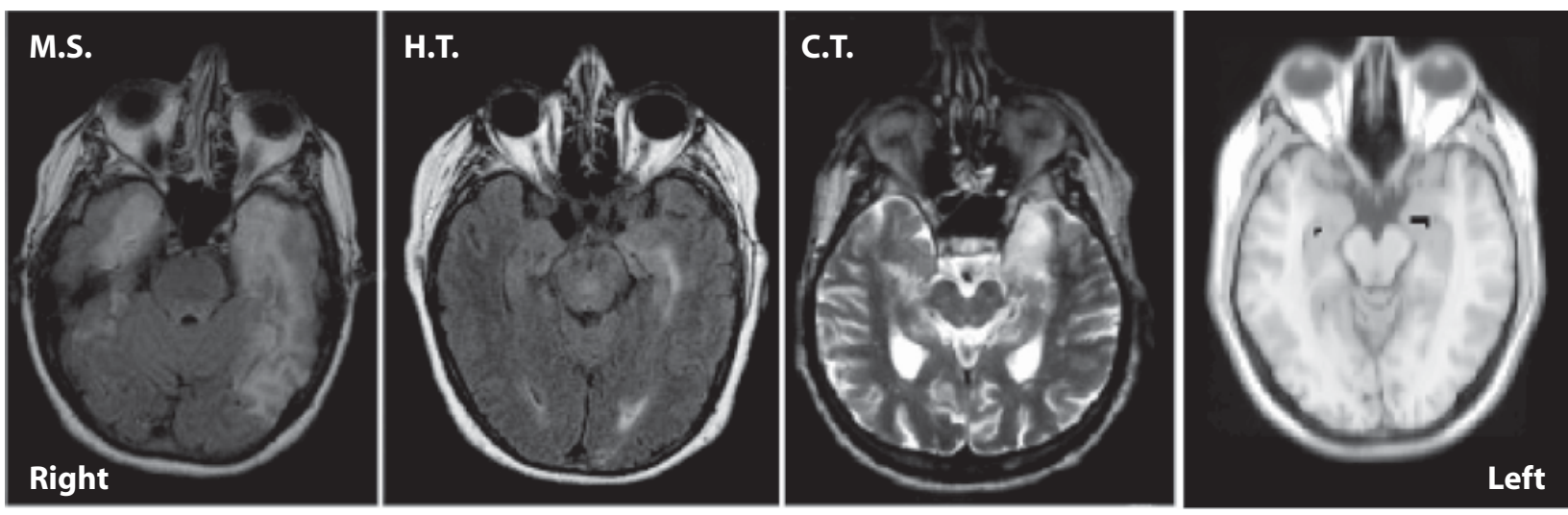

Figure 1. Axial MRI scans from the 3 patients-M.S., H.T., and C.T.-shown according to radiological convention (e.g., left on the right). For M.S. and H.T., fluid-attenuated inversion recovery (FLAIR) images are shown, and for C.T., T2-weighted images. The rightmost image was created with MRIcro and shows the extent of lesion overlap across patients, denoted in black.

many potential research issues, when viewed in light of the recent working memory findings. Specifically, there is still much to be learned about what role this region plays in linking working memory to LTM, with respect to how information is initially represented, maintained, and subsequently stored.

The primary goal of this study was to assess working memory over very short delays in patients with MTL damage. To extend prior studies of the MTL and working memory (Olson, Moore, et al., 2006; Olson, Page, et al., 2006), we used a parametric design in which we titrated the similarity of study and related lure stimuli, varied the delay period, and tested memory with both forced choice (FC) and old-new (ON) recognition tasks. Artificially generated human faces were used as stimuli because they have the benefit of being both salient and easy to parametrically manipulate along the dimension of similarity.

\section{METHOD}

\section{Participants}

The lesion group consisted of 3 patients with bilateral MTL damage (1 male, 2 females; 64-70 years of age, $M=67$ years; see Figure 1) due to encephalitis $(n=2)$ or to central nervous system (CNS) vasculitus. They had an average of 12 years of education and an average verbal IQ of 94, as measured by the Wechsler Adult Intelligence Scale, 3rd edition (WAIS; Wechsler, 1997a). Their mean General Memory and Visual Delayed Memory scores on the Wechsler Memory Scale, 3rd edition (Wechsler, 1997b) were 60 and 62, respectively. The only area of lesion overlap in the 3 patients was the anterior hippocampus (Figure 1, right panel). Detailed information about each patient follows.

Patient M.S. Patient M.S. (age 64) has bilateral MTL damage as a result of herpes encephalitis in 1999. The MTL damage extends into the amygdala, hippocampus, and perirhinal and parahippocampal cortex, on the left, and into the entorhinal cortex and hippocampus, on the right, as assessed by magnetic resonance imaging (MRI). Damage on the left also extends to posterior temporal regions. M.S.'s chief complaint is anomia, which has steadily lessened over time. When shown Snodgrass line drawings in 2002, she named only 16/65 correctly. Her anomia is most likely the result of left temporal pole damage (Lezak, 1995). Because the experiments reported in this article test visual memory, her naming difficulties were not a matter of concern. She did not self-report any visual problems, nor was there any evidence of vision problems when we asked her to match nonsense shapes on the
Mattis Dementia Rating Scale (Mattis, 1988), or when she was asked to match colors or faces. When asked to copy nonsense designs, she made only one small error; her line cancellation performance was errorless. She continues to drive on well-known streets and is actively involved in volunteer work in her community.

Patient H.T. Patient H.T. (age 66) has focal bilateral hippocampal damage, as evidenced by hypertensities in the hippocampus on T2-weighted MR scans (left greater than right), as well as a small hyperintensity in the left parietal lobe. The damage occurred in connection with basilar meningitis and CNS vasculitus. Her family reports that her behavior is unchanged from the past, except for a radical decline in her memory. She self-reported that she can no longer read novels or watch television, because she cannot follow the storylines. In addition, she sometimes gets confused when having a conversation, because of an inability to remember the topics. She has difficulty navigating and is not allowed to drive. Because her MRI showed a small left inferior parietal hyperintensity, her naming abilities were assessed with selected items from the Boston Naming Test, in which line drawings that vary from high to low frequency are presented; no deficit was found (7/8). She shows no neglect, acalculia, or other problems commonly associated with inferior parietal damage. Her reading was assessed by requiring her to read aloud 16 printed words; no deficit was found (15/16). She works part time as a receptionist at a health club.

Patient C.T. Patient C.T. (age 70) has MTL damage as a result of encephalitis experienced in 2001. His MRI scans show damage to the left anterior hippocampus, temporal pole, and portions of the entorhinal cortex, as well as more limited damage to the right anterior hippocampus. He self-reports that he can no longer navigate and gets lost in his own neighborhood. His naming abilities are intact, as shown by his Boston naming score of 58/60. Although he is officially retired, he continues to work as a skilled cabinetmaker.

Lesion overlap. MRIcro (www.sph.sc.edu/comd/rorden/mricro .html) was used to analyze lesion overlap among the patients (Rorden $\&$ Brett, 2000). All patients' lesions were drawn as regions of interest (ROIs) on a standard Montreal Neurological Institute brain. We found the intersection of these lesion ROIs using MRIcro and plotted the resulting overlap ROI.

Control group. The control participants were 10 older healthy adults ( 3 males, 7 females; 49-74 years of age, $M=57$ years) with an average of 13 years of education. Average verbal IQ, as measured by the WAIS (Wechsler, 1997a), was 112. There were no differences between the MTL lesion group and their control group in terms of age $(p>.15)$, education $(p>.25)$, or verbal IQ $(p>.05)$. All participants were cooperative and attentive and had normal or correctedto-normal visual acuity. All of them signed an informed consent form prior to taking part in the experiment. 


\section{Equipment}

The participants were tested individually on either a laptop or a desktop computer. They sat at an unrestricted viewing distance of about $57 \mathrm{~cm}$, at which distance $1 \mathrm{~cm}$ corresponds to $1^{\circ}$ of viewing angle. The experiment was programmed in E-Prime (www.pstnet .com/products/e-prime) for PC.

\section{Materials}

Morphed faces were created with GenHead 1.2 beta software (www.genemation.com). This software provides a database of highly realistic artificial faces that can be easily manipulated for experimental purposes. The stimuli were created as follows: 16 male and 16 female Caucasian faces (all dissimilar) were generated by GenHead. For any given gender, 2 of the 16 faces were selected to be the base pair for each set (denoted Base 1 and Base 2), and morphs were created by titrating the percentage of Base 2's face that was added to Base 1's face. This process continued until 32 morphed sets of faces had been produced, which were then used in the experiment. Each set consisted of Base 1 along with $40 \%$, $50 \%, 70 \%$, and $100 \%$ Base 2 faces. Figure 2 shows a representative base pair with the associated morphs. Faces were $105 \times 124 \mathrm{~mm}$ in size, at a resolution of 72 pixels/in. and $32 \mathrm{bits} /$ pixel, and were presented on a uniformly black background. A mask was created with a randomly chosen female face that was scrambled using Adobe Photoshop to ensure that the mask retained the color information present in the faces, but did not contain any features resembling those of an actual face.

\section{Design}

The dependent measures were accuracy and response time (RT). In addition to the between-subjects factor group (control or lesion), three within-subjects factors were manipulated: lure-target separa- tion (40\%, 50\%, 70\%, and 100\% different), lure side (left or right), and delay interval ( 1 or $8 \mathrm{sec}$ ). Lure side was not analyzed. All withinsubjects factors were randomly intermixed in the experiment.

\section{Procedure: FC Task}

The participants were tested on two tasks, an FC recognition memory task and an ON recognition memory task. Task order was counterbalanced across participants. Each trial started with an attentionorienting message ("Get Ready!") displayed for $0.5 \mathrm{sec}$, followed by a fixation " + " for $1 \mathrm{sec}$. A single female face was then presented for $1.5 \mathrm{sec}$ as the memory image. This was followed by a $100-\mathrm{msec}$ mask (to truncate visual processing and iconic memory) and a blank delay interval of either 1 or $8 \mathrm{sec}$. The memory image was always one of the base faces. Last, a probe image containing two female faces, side by side, was presented. The task was to decide which of the two probe faces matched the face presented in the memory image. Responses were made by unspeeded keypress. The probe display was then cleared and a mask presented for $100 \mathrm{msec}$, to truncate visual processing of the probe image faces before the next trial commenced. The testing session began with 16 practice trials using male faces, so that subjects had no prior exposure to the stimuli used in the task. Practice was followed by 96 randomly ordered test trials. Each base face served as a memory image on 6 trials. All of the lure-target combinations were presented equally often, for a total of 24 trials per lure-target combination. A sample trial is shown in Figure 2.

\section{Procedure: ON Task}

Each of these trials was identical to the FC trials, except that the probe image contained one female face in the center of the screen. The task was to decide whether the probe face matched the face presented in the memory image. Practice (16 trials) was followed by 96 randomly ordered test trials.
Base 1
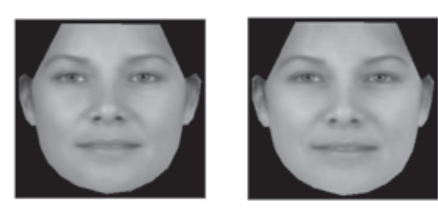

$50 \%$

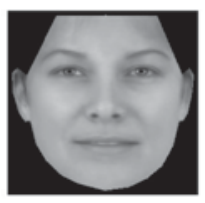

$70 \%$

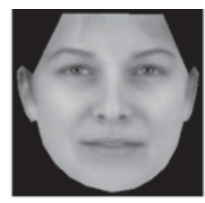

Base 2

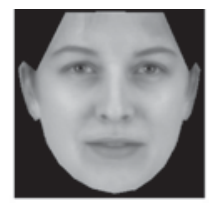

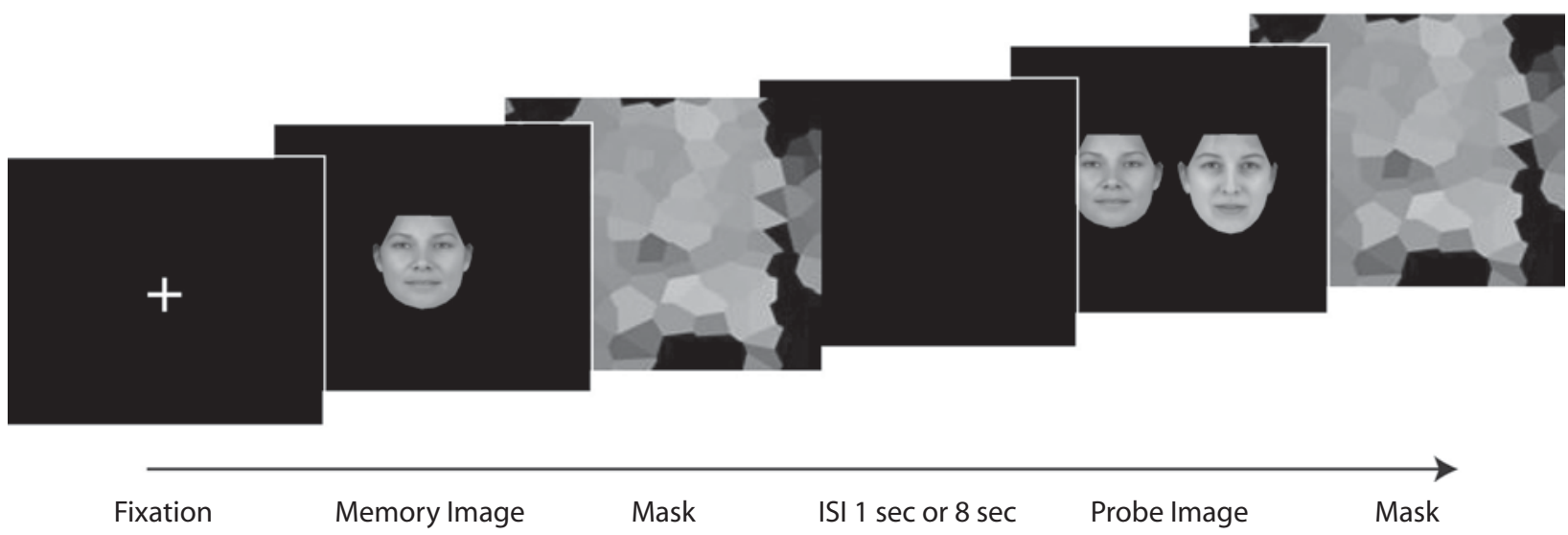

Figure 2. The top panels show a representative face set. Two female "base" faces (labeled Base 1 and Base 2) were used to create each set of morphed faces. The percentages refer to the amount of the Base 2 face present in each image. This morphing procedure was used to parametrically vary the similarity between studied and related-lure stimuli. The bottom panels show a schematic diagram of a single trial from the forced choice task. Each trial began with a "Get Ready!" message (500 msec), followed by a fixation cross (1 sec). A single face was then presented as the memory image $(1.5 \mathrm{sec})$. This was followed by a mask (100 msec) and a blank delay interval $(1 \mathrm{or} 8 \mathrm{sec})$. At last, a probe image containing the target and lure faces was presented until a response was made. The task was to decide which probe face had appeared in the memory image. In the example shown here, the lure-target separation is $100 \%$. The trial design for the old-new task was identical to that shown, except that a single face was displayed in the probe image, and the task was to decide whether the face matched the face from the memory image. 


\section{Forced Choice}

1 -sec Delay
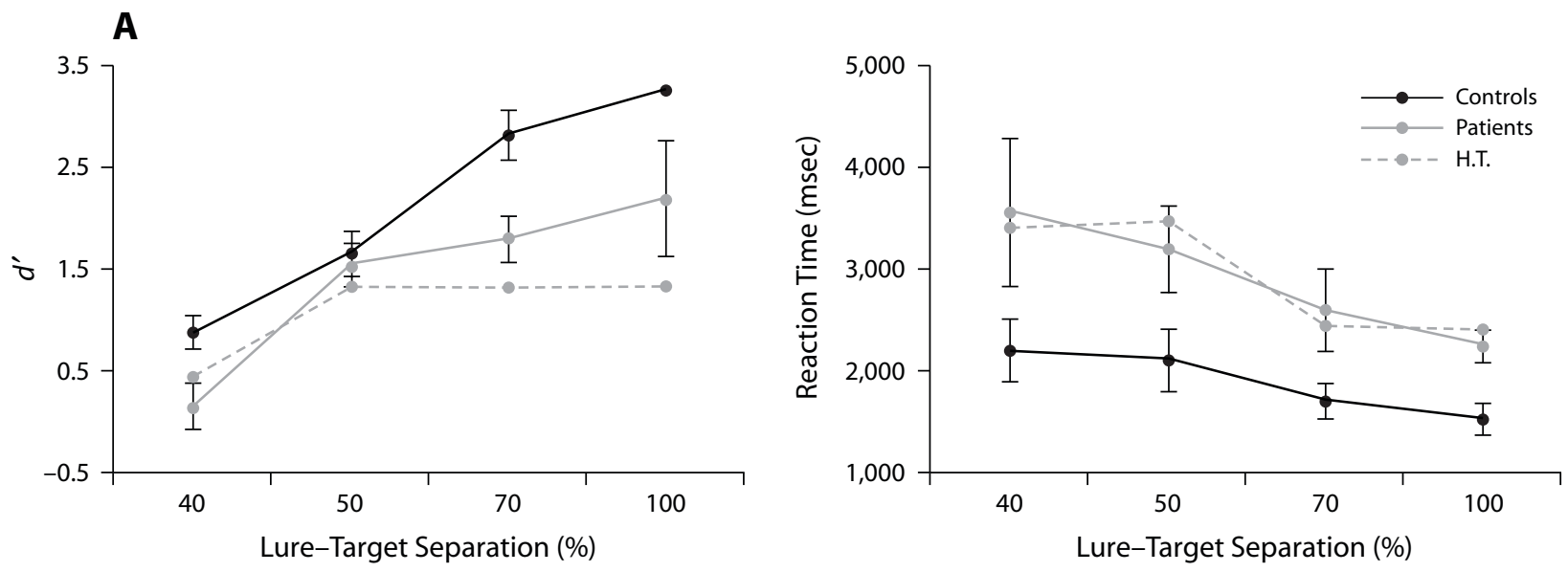

8-sec Delay

B

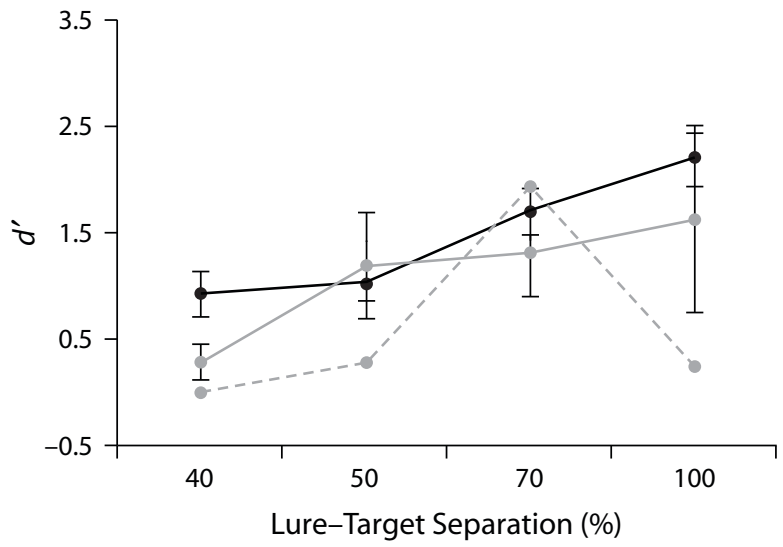

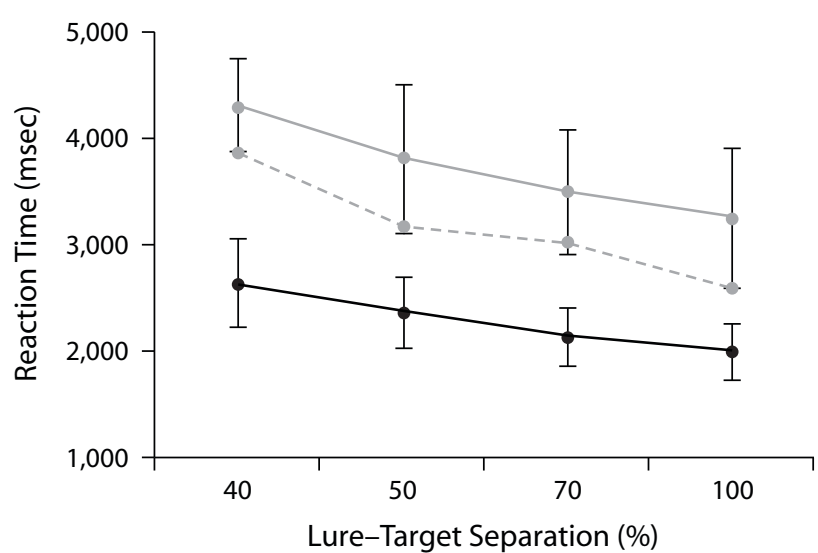

Figure 3. Mean $d^{\prime}$ scores (left column) and reaction times (right column) for amnesics and controls on the forced choice face working memory task at the (A) 1-sec and (B) 8-sec delays. Error bars represent 1 standard error of the mean. The dashed line on each graph represents the data from a single patient, H.T.

\section{Perception Test}

A perceptual analogue of the memory tasks was administered prior to the memory tasks. The participants were presented with a black computer screen divided in two halves, each half containing a face from the memory experiment. On half of the trials, the faces were identical. On the other half, the faces differed by $40 \%, 50 \%$, $70 \%$, or $100 \%$, just as in the memory task. The two trial types were randomly intermixed. The task was to look at the two faces and to make an unspeeded same-different judgment on the faces. After the response was entered, the screen cleared and the next trial commenced. There were 32 self-paced trials.

\section{Statistical Analysis}

Accuracy was converted to $d^{\prime}$ and analyzed using a repeated measures ANOVA. Incorrect trials were removed from the RT analysis, and the remaining trials were also analyzed using a repeated measures ANOVA. We note that, whereas the numbers of participants between the groups were unbalanced, the total replicate conditions yielded at least 10 degrees of freedom for within-group variance for each test, and the Shapiro-Wilks $W$ test for the residuals did not reject the assumption of normality.

\section{RESULTS}

\section{FC Task}

Figure 3 shows the results of the FC recognition task. Only statistics relevant to our hypotheses are reported. A repeated measures ANOVA on $d^{\prime}$ showed that amnesics were less accurate than the controls at remembering faces $\left[F(1,11)=6.02, p=.03\right.$; mean $d^{\prime}=1.28$ vs. 1.83$]$. Planned comparisons showed that the amnesics were less accurate at the 1 -sec delay $[t(11)=3.01, p=.006]$ but performed similarly to the controls at the 8 -sec delay $[t(11)=1.23, p=.12]$. Across groups and delays, performance suffered when the lure face was similar to the target face $\left[F(3,33)=28.78, p<.001 ;\right.$ mean $d^{\prime} \mathrm{s}=0.58$, $1.37,1.92$, and 2.34]. In addition, overall performance was worse at the longer delay interval $[F(1,11)=10.79$, $p=.007$; mean $d^{\prime}=1.30$ vs. 1.80$]$. The interactions of group $\times$ similarity and group $\times$ similarity $\times$ delay did 
not provide evidence of differentially poor performance on trials with similar lures and targets (all $p \mathrm{~s}>.15$ ).

The results of the RT analysis (see Figure 3, right column) showed that amnesics were on average 1,224 msec slower than controls $[F(1,11)=4.87, p=.05]$. Planned comparisons showed that amnesics were slower at both the $1-\sec [t(11)=2.15, p=.03]$ and $8-\sec [t(11)=2.20, p=$ $.03]$ delays. Across groups and delays, performance was slower when the lure face was similar to the target face $[F(3,33)=19.97, p<.001]$. In addition, overall performance was on average $495 \mathrm{msec}$ slower at the longer delay interval $[F(1,11)=20.77, p<.001]$. The interactions of group $\times$ similarity and group $\times$ similarity $\times$ delay did not provide evidence of differentially slower performance on trials with similar lures and targets (all $p \mathrm{~s}>.20$ ).

These findings suggest that amnesics have impaired visual working memory at 1 -sec delays, as evidenced by lower accuracy and slower responses. Impairments were also observed at the 8-sec delay, but only in the RT measure. It is likely that impairments were not observed in the accuracy measure because both groups performed very poorly at the 8-sec delay, with the amnesics showing floor effects. However, amnesics were not differentially impaired when lures and targets were similar.

\section{ON Task}

Figure 4 shows the results of the ON recognition task. A repeated measures ANOVA on $d^{\prime}$ showed that amnesics were less accurate than the controls at remembering faces $\left[F(1,11)=4.51, p=.057\right.$; mean $d^{\prime}=1.71 \mathrm{vs}$. 3.50]. Planned comparisons showed that amnesics were marginally less accurate than the controls at the 1-sec delay $[t(11)=1.58, p=.07]$ but were significantly less accurate at the 8 -sec delay $[t(11)=2.05, p=.03]$. Across groups

\section{Old-New}

1-sec Delay
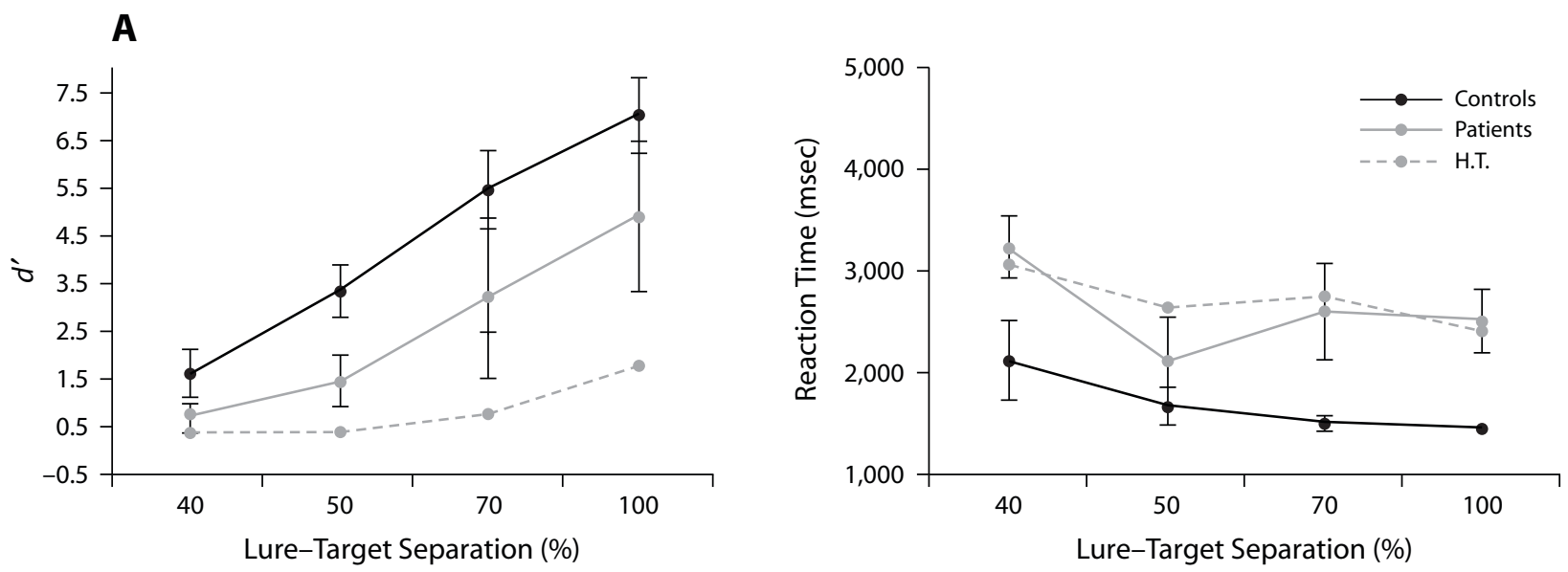

8-sec Delay

B

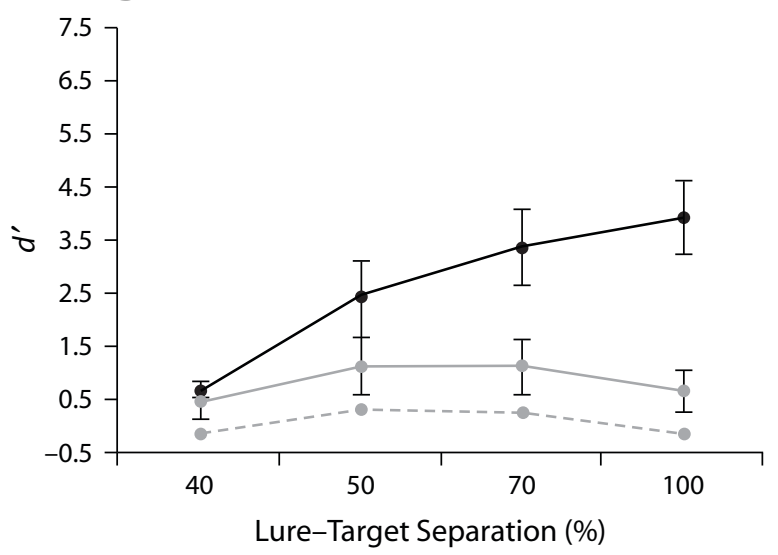

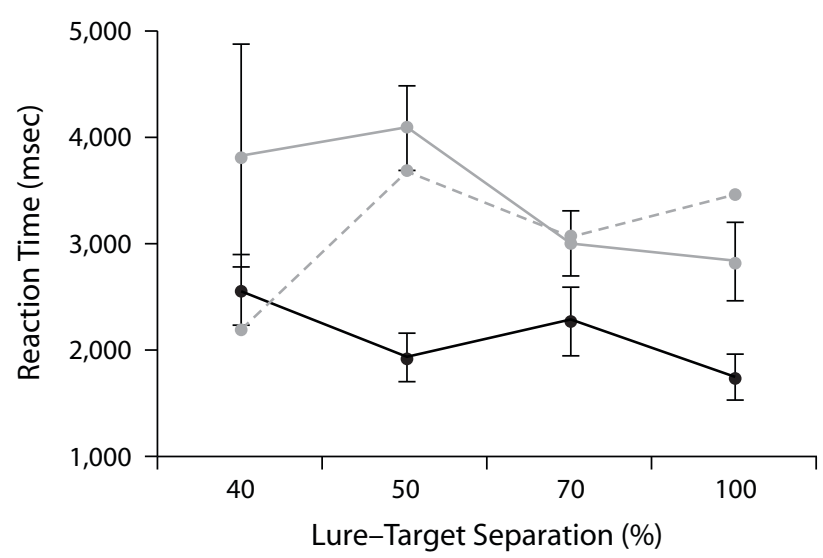

Figure 4. Mean $d^{\prime}$ scores (left column) and reaction times (right column) for amnesics and controls on the old-new face working memory task at the (A) 1-sec and (B) 8-sec delays. Error bars represent 1 standard error of the mean. The dashed line on each graph represents the data from a single patient, H.T. 
and delays, performance suffered when the lure face was similar to the target face $[F(3,33)=16.34, p<.001 ;$ mean $d^{\prime} \mathrm{s}=0.88,2.09,3.30$, and 4.14]. In addition, overall performance was worse at the longer delay interval $[F(1,11)=$ $9.41, p=.01$; mean $d^{\prime}=1.74$ vs. 3.47$]$. The interactions of group $\times$ similarity and group $\times$ similarity $\times$ delay did not provide evidence of differentially poor performance on trials with similar lures and targets (all $p \mathrm{~s}>.15$ ).

The results of the RT analysis (see Figure 4, right column) showed that amnesics were on average 1,093 msec slower than the controls $[F(1,10)=6.73, p<.03]$. Planned comparisons showed that amnesics were slower at both the $1-\sec [t(11)=2.64, p=.01]$ and $8-\sec [t(11)=2.58, p=$ $.01]$ delays. Across groups and delays, performance was slower when the lure face was similar to the target face $[F(3,30)=5.35, p=.005]$. In addition, overall performance was on average $667 \mathrm{msec}$ slower at the longer delay interval $[F(1,10)=14.50, p=.003]$. The interaction of group $\times$ similarity provided no evidence of differentially poor performance on trials with similar lures and targets $(F<1$, n.s. $)$. However, the interaction of group $\times$ similarity $\times$ delay provided evidence that the patients were differentially slowed on trials with dissimilar lures and targets at the 8 -sec delay $[F(3,30)=3.68, p<.03]$. In other words, amnesics did not have improved performance at the 8-sec delay when the recall task made the trial easier.

These findings show that on the ON recognition task, amnesics' performance was partially intact at the 1 -sec delay, since impairments were observed primarily on the RT measure. At the 8-sec delay, however, the amnesics' performance was impaired on both measures. It was not differentially impaired, though, when lures and targets were similar. Rather, they were differentially impaired when lures and targets were dissimilar at the 8-sec delay (see Figure 4).

\section{Task Effects}

To assess whether amnesics were impaired on one task as compared with the other, a repeated measures ANOVA with group and task as factors was conducted, collapsing across other factors. The results showed that, overall, amnesics were less accurate $[F(1,11)=5.56, p=.04$; mean $d^{\prime}=1.50$ vs. 2.66$]$ and slower $[F(1,11)=7.03, p=.02$; mean RT $=3,177$ vs. 2,002 msec] than controls. Both groups were less accurate on the FC task than on the $\mathrm{ON}$ task $\left[F(1,11)=8.30, p=.02\right.$; mean $d^{\prime}=1.55$ vs. 2.61$]$, though there were no speed differences $[F(1,11)=1.55$, $p=.24]$. The amnesics did not numerically appear to be worse than controls on the ON task, and the interaction of task $\times$ group provided no evidence of a statistical difference $(p>.10)$.

\section{Perception Task}

A repeated measures ANOVA found that the groups did not differ in accuracy $(F<1$, n.s. $)$ or speed $[F(1,10)=$ $3.06, p=.11]$ on the perceptual control task. Because patient M.S.'s lesion extended into perirhinal and parahippocampal cortex, we also compared her with controls separately and found no difference for accuracy $[F(1,9)=$ $1.43, p=.26]$, although she was slower overall $[F(1,9)=$
$11.91, p=.007$; mean RT $=11,859$ vs. $4,934 \mathrm{msec}]$. To see whether her slower responses on the perception task had any bearing on the working memory task, we compared her with the other 2 patients. She did not differ from them significantly in terms of accuracy or RT on the FC and $\mathrm{ON}$ tasks (all $p \mathrm{~s}>.17$ ).

\section{DISCUSSION}

The role of the MTL memory system in working memory is controversial. The paradigmatic role of the MTL, or (more precisely) the hippocampus, is in LTM, with no role in short-term memory processes (Baddeley \& Warrington, 1970; Scoville \& Milner, 1957). However, recent findings from our lab (Olson, Moore, et al., 2006; Olson, Page, et al., 2006) and others (Hannula, Tranel, \& Cohen, 2006; Hartley et al., 2007; Ranganath \& D'Esposito, 2005) have called into question this dichotomy. The findings detailed in this article provide evidence that the human MTL is critical for accurate visual working memory. Impaired visual working memory was not associated with any particular recollection task, since amnesics were impaired on both the forced choice and old-new recognition procedures. Results from the FC task show that amnesics were impaired at the 1-sec delay on both accuracy and RT measures. At the 8-sec delay, impairments were only observed in the RT measures, whereas accuracy was compromised by floor effects. Results from the ON task showed that amnesics were slower, but still accurate at the 1-sec delay. However, at the 8-sec delay, impairments were observed on both accuracy and RT measures. Although overall performance was lower on the FC task, amnesics were not disproportionately impaired on one task or the other. These findings suggest that the human MTL is critical for accurate visual working memory or short-term memory.

\section{Visual Working Memory and the Hippocampus}

Our findings replicate and extend other studies of face working memory using $\mathrm{ON}$ recognition procedures and accuracy measures over delays of $4 \mathrm{sec}$ (Olson, Moore, et al., 2006) or $7 \mathrm{sec}$ (Nichols, Kao, Verfaellie, \& Gabrieli, 2006). Other recent studies have reported that hippocampal damage causes working memory impairments for colors, locations (Olson, Moore, et al., 2006), objectlocation conjunctions (Olson, Page, et al., 2006), facescene conjunctions (Hannula et al., 2006), and conjunctions between items in a scene (Ryan \& Cohen, 2004).

As long ago as 1973, it was reported that MTL amnesics had impaired memory for faces over delays of less than a few seconds (Warrington \& Taylor, 1973). It was suggested that this reflected a long-term, not a short-term, memory deficit because there was no evidence of a decay function over a 30 -sec period of time, and decay was believed to be a signature of short-term forms of memory. In contrast, we found that face memory decayed between the 1- and 8-sec delays tested in our experiment. Other authors have suggested that face or object memory impairments found in amnesics at delays as short as $6 \mathrm{sec}$ reflect the rapid recruitment of LTM (Buffalo, Reber, \& Squire, 1998). Similar suggestions have been offered within the neuroimaging 
literature to explain hippocampal activations during visual working memory tasks (Ranganath \& D'Esposito, 2005; Schon, Hasselmo, LoPresti, Tricarico, \& Stern, 2004; Stern, Sherman, Kirchhoff, \& Hasselmo, 2001).

The difficulty in interpreting the relationship between the hippocampus and short-term forms of memory when no clear behavioral indices can demark the two memory processes prompts consideration of whether visual working memory can be clearly distinguished from visual LTM. The representational format of information held in visual working memory and LTM is similar (Hollingworth, 2004), and oft-touted capacity differences between working memory and LTM may be due to differences in testing format that lead to precision/capacity trade-offs. It is possible that visual memory is different from verbal memory, in that fewer mechanisms exist for visual than for verbal rehearsal. This could reflect qualitative differences between visual and verbal memory; perhaps visual memory relies on a single-store (Nairne, 2002) rather than a dual-store memory system.

The idea of a dual-store memory system has long influenced the theory and practice of memory research (Baddeley, 2003). This distinction has proven useful in many instances and has also helped cement the view that the MTL is involved exclusively in LTM processes. The research that has fueled this distinction is overwhelmingly from studies of the phonological loop (Baddeley, 2003). In comparison, the visual portion of working memory, or the visuospatial sketchpad, has played only a small role in the theoretical development of working memory. Recent findings, from our laboratory and others, have suggested that we may need to update our theoretical understanding of working memory. Now that it has become clear that working memory and LTM share neural space in the MTL, precisely characterizing the relationship between the two should be an exciting focus of future studies.

\section{Encoding and the Hippocampus}

The mechanism by which the hippocampus encodes memories is not fully understood. Computational models have proposed that changes in synaptic strength (Hasselmo \& Schnell, 1994; Treves \& Rolls, 1992) or timing relative to the theta rhythm (Hasselmo, Bodelón, \& Wyble, 2002; Kunec, Hasselmo, \& Kopell, 2005) allow for the encoding of associations. The CA3 subfield of the hippocampus has been targeted as specifically involved in encoding, since it receives input directly from the entorhinal cortex and indirectly from the dentate gyrus, and it is equipped to perform autoassociative functions (Amaral \& Witter, 1989; Kunec et al., 2005).

Here, we attempted to examine encoding processes in the hippocampus by using short delay intervals. By assessing memory so quickly after the stimulus was presented, we specifically targeted stimulus information that was relayed to and encoded by the hippocampus. In this sense, we did not examine encoding in terms of consolidation, but rather in terms of how the stimuli were represented in the hippocampus and MTL shortly after presentation. This kind of "representational" question has been touched on previously in computational models (Norman \& O'Reilly,
2003) but has rarely been considered behaviorally. Although the procedure used in the present study is likely not ideal for addressing these "representational" questions, it is nonetheless an important step toward properly defining the relationship between LTM and working memory.

\section{Effects of Damage Outside the Hippocampus: Perirhinal and Parietal Cortices}

Recently, debate over the role of the perirhinal cortex in perception and memory has intensified. Although traditionally viewed as an area important for mnemonic processing (Squire \& Zola-Morgan, 1991), recent work has raised the possibility that the perirhinal cortex may play some role in high-level perception, distinct from its role in memory (Buckley, Booth, Rolls, \& Gaffan, 2001; Bussey, Saksida, \& Murray, 2002; Murray \& Bussey, 1999). The fact that patients C.T. and M.S. have lesions extending outside the hippocampus, encompassing the perirhinal cortex, prompts a discussion of whether perceptual deficits created by perirhinal damage account for the differences observed between our patient and control groups. There are two reasons that we rule out perceptual explanations for our results.

First, primate studies have reported impaired perception primarily in instances in which a large number of highly similar stimuli were to be discriminated (reviewed in Buckley \& Gaffan, 2006; Bussey, Saksida, \& Murray, 2006), raising the question of whether visual memory deficits lie at the heart of the observed impairments. Indeed, perceptual deficits in monkeys are most often observed in tasks with a significant learning or memory component (Hampton, 2005). The human literature on this topic does not help to resolve this question, because perceptual and mnemonic processes were confounded in some experiments, and in all cases, sample populations also had significant damage to the hippocampus and gross disturbances of memory (Barense et al., 2005; Lee, Barense, \& Graham, 2005; Lee et al., 2006; Lee, Bussey, et al., 2005). In sum, the claim that perirhinal damage impairs perception but not memory is controversial and is supported by some studies - noted above- - but not others (Buffalo et al., 1998; Hampton, 2005; Holdstock, Gutnikov, Gaffan, \& Mayes, 2000; Levy, Shrager, \& Squire, 2005; Stark $\&$ Squire, 2000). In the best case, when perceptual deficits are observed, they are associated with tasks that require the comparison of many items simultaneously and, typically, some learning or memory demand-circumstances not found in the present experiment.

Second, our patients with more extensive lesions performed similarly to our patient with focal hippocampal damage on the face working memory tests. The patient group also showed no difference from the control group on the perceptual control task. For these reasons, we believe it unlikely that the types of perceptual deficits that have been attributed to perirhinal damage contribute in a meaningful way to the results reported in this article.

In addition, we must also consider the possibility that the small hyperintensity in the white matter of the left parietal lobe of patient H.T. affected her visual working memory performance. Several neuroimaging studies have reported activations along the intraparietal sulcus to various visual 
working memory tasks (e.g., Todd \& Marois, 2004; Xu \& Chun, 2006). However, evidence from neuropsychology is less conclusive on this point, with the most significant finding linking verbal working memory impairments to left parietal lobe damage (Fiez, 2001). Given that our patient showed no classic symptoms of left parietal damage (e.g., acalculia, apraxia, aphasia, or neglect), did not have damage to the intraparietal sulcus, and was not tested on any verbal working memory tasks, we do not believe that the left parietal damage is of concern in this study.

\section{Conclusions}

The present study describes the cases of 3 patients in which MTL damage caused impaired working memory for faces over delays of 1 and $8 \mathrm{sec}$. Deficits were found on both forced choice and old-new recognition tasks and on both accuracy and RT measures. In addition, memory on both high- and low-similarity trials was impaired in MTL amnesics. These findings support the recent idea that this region is critical for accurate visual working memory, which, combined with the well-established consensus that MTL is critical for LTM, suggests that this area may be important in processing working memory for the long term. Our results raise many issues that relate to memory and the MTL, and thus provide several possibilities for expansion in future research.

\section{AUTHOR NOTE}

We thank Geoff Aguirre and Amy Thomas for providing stimuli, Marianna Stark for recruiting test participants, and Anjan Chatterjee for helpful advice. We also thank our patient group, who generously donated their time. This research was supported in part by NIMH Grant RO1 MH071615 to I.R.O. Correspondence relating to this article may be sent to I. R. Olson, Department of Psychology, Temple University, 1701 13th Street, Philadelphia, PA 19122 (e-mail: iolson@temple.edu).

Note-This article was accepted by the previous editorial team, when John Jonides was Editor.

\section{REFERENCES}

Amaral, D. G., \& Witter, M. P. (1989). The three-dimensional organization of the hippocampal formation: A review of anatomical data. Neuroscience, 31, 571-591.

BADDELEY, A. [D.] (2003). Working memory: Looking back and looking forward. Nature Reviews Neuroscience, 4, 829-839.

Baddeley, A. D., \& Warrington, E. K. (1970). Amnesia and the distinction between long- and short-term memory. Journal of Verbal Learning \& Verbal Behavior, 9, 176-189.

Barense, M. D., Bussey, T. J., Lee, A. C. H., Rogers, T. T., Davies, R. R., SAKSIDA, L. M., ET AL. (2005). Functional specialization in the human medial temporal lobe. Journal of Neuroscience, 25, 10239-10246.

Buckley, M. J., Booth, M. C. A., Rolls, E. T., \& Gaffan, D. (2001). Selective perceptual impairments after perirhinal cortex ablation. Journal of Neuroscience, 21, 9824-9836.

Buckley, M. J., \& GAFFAN, D. (2006). Perirhinal cortical contributions to object perception. Trends in Cognitive Sciences, 10, 100-107.

Buffalo, E. A., Reber, P. J., \& SQuire, L. R. (1998). The human perirhinal cortex and recognition memory. Hippocampus, 8, 330-339.

Bussey, T. J., Saksida, L. M., \& Murray, E. A. (2002). Perirhinal cortex resolves feature ambiguity in complex visual discriminations. European Journal of Neuroscience, 15, 365-374.

Bussey, T. J., Saksida, L. M., \& Murray, E. A. (2006). Perirhinal cortex and feature-ambiguous discriminations. Learning \& Memory, 13, 103-105.

DaVachi, L., Mitchell, J. P., \& Wagner, A. D. (2003). Multiple routes to memory: Distinct medial temporal lobe processes build item and source memories. Proceedings of the National Academy of Sciences, 100, 2157-2162.

Day, M., Langston, R., \& Morris, R. G. M. (2003). Glutamatereceptor-mediated encoding and retrieval of paired-associate learning. Nature, 424, 205-209.

Dobbins, I. G., Rice, H. J., Wagner, A. D., \& Schacter, D. L. (2003). Memory orientation and success: Separable neurocognitive components underlying episodic recognition. Neuropsychologia, 41, 318-333.

Düzel, E., Habib, R., Rotte, M., Guderian, S., Tulving, E., \& HeInZE, H.-J. (2003). Human hippocampal and parahippocampal activity during visual associative recognition memory for spatial and nonspatial stimulus configurations. Journal of Neuroscience, 23, 9439-9444.

Eldridge, L. L., Knowlton, B. J., Furmanski, C. S., Bookheimer, S. Y., $\&$ ENGEL, S. A. (2000). Remembering episodes: A selective role for the hippocampus during retrieval. Nature Neuroscience, 3, 1149-1152.

FIEZ, J. A. (2001). Bridging the gap between neuroimaging and neuropsychology: Using working memory as a case-study. Journal of Clinical \& Experimental Neuropsychology, 23, 19-31.

HAMPTON, R. R. (2005). Monkey perirhinal cortex is critical for visual memory, but not for visual perception: Reexamination of the behavioural evidence from monkeys. Quarterly Journal of Experimental Psychology, 58B, 283-299.

Hannula, D. E., Tranel, D., \& Cohen, N. J. (2006). The long and the short of it: Relational memory impairments in amnesia, even at short delays. Journal of Neuroscience, 26, 8352-8359.

Hartley, T., Bird, C. M., Chan, D., Cipolotti, L., Husain, M., Vargha-KhadeM, F., \& Burgess, N. (2007). The hippocampus is required for short-term topographical memory in humans. Hippocampus, 17, 34-48.

Hasselmo, M. E., Bodelón, C., \& Wyble, B. P. (2002). A proposed function for hippocampal theta rhythm: Separate phases of encoding and retrieval enhance reversal of prior learning. Neural Computation, 14, 793-817.

Hasselmo, M. E., \& Schnell, E. (1994). Laminar selectivity of the cholinergic suppression of synaptic transmission in rat hippocampal region CA1: Computational modeling and brain slice physiology. Journal of Neuroscience, 14, 3898-3914.

Holdstock, J. S., Gutnikov, S. A., Gaffan, D., \& Mayes, A. R. (2000). Perceptual and mnemonic matching-to-sample in humans: Contributions of the hippocampus, perirhinal and other medial temporal lobe cortices. Cortex, 36, 301-322.

HollingwORTH, A. (2004). Constructing visual representations of natural scenes: The roles of short- and long-term visual memory. Journal of Experimental Psychology: Human Perception \& Performance, 30, 519-537.

JACKSON, O., III, \& SchaCter, D. L. (2004). Encoding activity in anterior medial temporal lobe supports subsequent associative recognition. NeuroImage, 21, 456-462.

JARRARD, L. E., DAVIDSON, T. L., \& Bowring, B. (2004). Functional differentiation within the medial temporal lobe in the rat. Hippocampus, 14, 434-449.

Kesner, R. P., \& Hopkins, R. O. (2006). Mnemonic functions of the hippocampus: A comparison between animals and humans. Biological Psychology, 73, 3-18.

Kunec, S., Hasselmo, M. E., \& Kopell, N. (2005). Encoding and retrieval in the CA3 region of the hippocampus: A model of theta-phase separation. Journal of Neurophysiology, 94, 70-82.

Lee, A. C. H., Barense, M. D., \& Graham, K. S. (2005). The contribution of the human medial temporal lobe to perception: Bridging the gap between animal and human studies. Quarterly Journal of Experimental Psychology, 58B, 300-325.

Lee, A. C. H., Buckley, M. J., Gaffan, D., Emery, T., Hodges, J. R., \& Graham, K. S. (2006). Differentiating the roles of the hippocampus and perirhinal cortex in processes beyond long-term declarative memory: A double dissociation in dementia. Journal of Neuroscience, 26, 5198-5203.

Lee, A. C. H., Bussey, T. J., Murray, E. A., Saksida, L. M., EpStein, R. A., KapUr, N., ET AL. (2005). Perceptual deficits in amnesia: Challenging the medial temporal lobe "mnemonic" view. Neuropsychologia, 43, 1-11.

LePage, M., Habib, R., \& Tulving, E. (1998). Hippocampal PET ac- 
tivations of memory encoding and retrieval: The HIPER model. Hippocampus, $\mathbf{8}, 313-322$.

Levy, D. A., Shrager, Y., \& SQuire, L. R. (2005). Intact visual discrimination of complex and feature-ambiguous stimuli in the absence of perirhinal cortex. Learning \& Memory, 12, 61-66.

LEZAK, M. D. (1995). Neuropsychological assessment (3rd ed.). New York: Oxford University Press.

Mattis, S. (1988). Dementia Rating Scale-2 (DRS-2). Odessa, FL: Psychological Assessment Resources.

Murray, E. A., \& Bussey, T. J. (1999). Perceptual-mnemonic functions of the perirhinal cortex. Trends in Cognitive Sciences, 3, 142-151.

NAIRNE, J. S. (2002). Remembering over the short-term: The case against the standard model. Annual Review of Psychology, 53, 53-81.

Nichols, E. A., Kao, Y.-C., Verfaellie, M., \& Gabrieli, J. D. E. (2006). Working memory and long-term memory for faces: Evidence from fMRI and global amnesia for involvement of the medial temporal lobes. Hippocampus, 16, 604-616.

Norman, K. A., \& O'Reilly, R. C. (2003). Modeling hippocampal and neocortical contributions to recognition memory: A complementarylearning-systems approach. Psychological Review, 110, 611-646.

Olson, I. R., Moore, K. S., Stark, M., \& ChatterJee, A. (2006). Visual working memory is impaired when the medial temporal lobe is damaged. Journal of Cognitive Neuroscience, 18, 1087-1097.

Olson, I. R., Page, K., Moore, K. S., Chatterjee, A., \& VerfaelLIE, M. (2006). Working memory for conjunctions relies on the medial temporal lobe. Journal of Neuroscience, 26, 4596-4601.

O'Reilly, R. C., \& McClelland, J. L. (1994). Hippocampal conjunctive encoding, storage, and recall: Avoiding a trade-off. Hippocampus, 4, 661-682.

O'Reilly, R. C., \& Norman, K. A. (2002). Hippocampal and neocortical contributions to memory: Advances in the complementary learning systems framework. Trends in Cognitive Sciences, 6, 505-510.

PihlajamäKi, M., Tanila, H., Hänninen, T., Könönen, M., MikKONEN, M., JALKANEN, V., ET AL. (2003). Encoding of novel picture pairs activates the perirhinal cortex: An fMRI study. Hippocampus, 13, 67-80

Pihlajamäki, M., Tanila, H., Könönen, M., Hänninen, T., HämäLÄInen, A., SoIninen, H., \& Aronen, H. J. (2004). Visual presentation of novel objects and new spatial arrangements of objects differentially activates the medial temporal lobe in subareas in humans. European Journal of Neuroscience, 19, 1939-1949.

Preston, A. R., Shrager, Y., Dudukovic, N. M., \& Gabrieli, J. D. E. (2004). Hippocampal contribution to the novel use of relational information in declarative memory. Hippocampus, 14, 148-152.

Ranganath, C., Cohen, M. X., \& Brozinsky, C. J. (2005). Working memory maintenance contributes to long-term memory formation: Neural and behavioral evidence. Journal of Cognitive Neuroscience, 17, 994-1010.

Ranganath, C., \& D'Esposito, M. (2005). Directing the mind's eye: Prefrontal, inferior and medial temporal mechanisms for visual working memory. Current Opinion in Neurobiology, 15, 175-182.

Rolls, E. T. (1996). A theory of hippocampal function in memory. Hippocampus, 6, 601-620.

Rorden, C., \& BRett, M. (2000). Stereotaxic display of brain lesions. Behavioural Neurology, 12, 191-200.

Ryan, J. D., \& CohEn, N. J. (2004). The nature of change detection and online representations of scenes. Journal of Experimental Psychology: Human Perception \& Performance, 30, 988-1015.

SCHACTER, D. L., \& WAGNER, A. D. (1999). Medial temporal lobe activations in fMRI and PET studies of episodic encoding and retrieval. Hippocampus, 9, 7-24.
Schon, K., Hasselmo, M. E., LoPresti, M. L., Tricarico, M. D., \& STERN, C. E. (2004). Persistence of parahippocampal representation in the absence of stimulus input enhances long-term encoding: A functional magnetic resonance imaging study of subsequent memory after a delayed match-to-sample task. Journal of Neuroscience, 24, 11088-11097.

Scoville, W. B., \& Milner, B. (1957). Loss of recent memory after bilateral hippocampal lesions. Journal of Neurology, Neurosurgery \& Psychiatry, 20, 11-21.

Small, S. A., Nava, A. S., Perera, G. M., DelaPaz, R., Mayeux, R., \& STERn, Y. (2001). Circuit mechanisms underlying memory encoding and retrieval in the long axis of the hippocampal formation. Nature Neuroscience, 4, 442-449.

Squire, L. R., \& Zola-Morgan, S. (1991). The medial temporal lobe memory system. Science, 253, 1380-1386.

Stark, C. E. L., \& SQuire, L. R. (2000). Intact visual perceptual discrimination in humans in the absence of perirhinal cortex. Learning \& Memory, 7, 273-278.

Stark, C. E. L., \& SQuire, L. R. (2001). Simple and associative recognition memory in the hippocampal region. Learning \& Memory, 8, 190-197.

Stern, C. E., Sherman, S. J., Kirchhoff, B. A., \& Hasselmo, M. E. (2001). Medial temporal and prefrontal contributions to working memory tasks with novel and familiar stimuli. Hippocampus, 11, 337-346.

Strange, B. A., Fletcher, P. C., Henson, R. N., Friston, K. J., \& Dolan, R. J. (1999). Segregating the functions of human hippocampus. Proceedings of the National Academy of Sciences, 96, 4034-4039.

Strange, B. A., Otten, L. J., Josephs, O., RugG, M. D., \& Dolan, R. J. (2002). Dissociable human perirhinal, hippocampal, and parahippocampal roles during verbal encoding. Journal of Neuroscience, 22, 523-528.

TodD, J. J., \& Marois, R. (2004). Capacity limit of visual short-term memory in human posterior parietal cortex. Nature, 428, 751-754.

Treves, A., \& Rolls, E. T. (1992). Computational constraints suggest the need for two distinct input systems to the hippocampal CA3 network. Hippocampus, 2, 189-199.

WARRINGTON, E. K., \& TAYLOR, A. M. (1973). Immediate memory for faces: Long- or short-term memory? Quarterly Journal of Experimental Psychology, 25, 316-322.

WECHSLER, D. (1997a). Wechsler Adult Intelligence Scale, third edition: Administration and scoring manual. San Antonio, TX: Psychological Corp.

WeCHSLER, D. (1997b). Wechsler Memory Scale, third edition: Administration and scoring manual. San Antonio, TX: Psychological Corp.

Xu, Y., \& ChUn, M. M. (2006). Dissociable neural mechanisms supporting visual short-term memory for objects. Nature, 440, 91-95.

Yonelinas, A. P. (2002). The nature of recollection and familiarity: A review of 30 years of research. Journal of Memory \& Language, 46, 441-517.

Yonelinas, A. P., Kroll, N. E. A., Quamme, J. R., Lazzara, M. M., Sauvé, M.-J., Widaman, K. F., \& Knight, R. T. (2002). Effects of extensive temporal lobe damage or mild hypoxia on recollection and familiarity. Nature Neuroscience, 5, 1236-1241.

Zeineh, M. M., Engel, S. A., Thompson, P. M., \& Bookheimer, S. Y. (2003). Dynamics of the hippocampus during encoding and retrieval of face-name pairs. Science, 299, 577-580.

(Manuscript received January 20, 2007; revision accepted for publication April 25, 2007.) 